\title{
Impacts of viscous dissipation and Joule heating on hydromagnetic boundary layer flow of nanofluids over a flat surface subjected to Newtonian heating
}

\author{
Santosh Chaudhary ${ }^{1} \cdot \mathrm{KM}_{\text {Kanika }}{ }^{1}$
}

Received: 29 July 2019 / Accepted: 16 November 2019 / Published online: 28 November 2019

(c) Springer Nature Switzerland AG 2019

\begin{abstract}
Main concern in this analysis is to study the two-dimensional, steady boundary layer flow of viscous, incompressible, electrically conducting nanofluids past a flat plate in the presence of magnetic field with heat being transferred by Newtonian heating way. Influences of viscous dissipation and Joule heating are considered also. Nonlinear partial differential equations are reduced into nonlinear ordinary differential equations by formulating similarity transformations. Numerical solutions of transformed boundary layer equations are clarified by applying the Keller-Box method. Effects of several types of nanofluids and various specified parameters such as solid volume fraction, magnetic parameter, Brinkmann number and local Biot number on velocity and temperature fields have been plotted graphically, while values of surface shear stress and surface heat flux are presented via table. Further, comparison of obtained computational values has been made with earlier published results for non-magnetic case.
\end{abstract}

Keywords Viscous dissipation · Joule heating · Hydromagnetic boundary layer flow · Nanofluids · Flat surface · Newtonian heating

$\begin{array}{ll}\text { List of symbols } \\ B_{0} \quad \text { Uniform magnetic field strength }\left(\mathrm{N} \mathrm{m}^{-1} \mathrm{~A}^{-1}\right) \\ B i & \text { Local Biot number } \\ B r & \text { Brinkmann number } \\ C_{f} & \text { Local skin friction coefficient } \\ C_{p} & \text { Specific heat at constant pressure }\left(\mathrm{J} \mathrm{Kg}^{-1} \mathrm{~K}^{-1}\right) \\ f & \text { Dimensionless stream function } \\ h_{t} & \text { Heat transfer coefficient } \\ M & \text { Magnetic parameter } \\ N u_{x} & \text { Local Nusselt number } \\ P r & \text { Prandtl number } \\ R e_{x} & \text { Local Reynolds number } \\ T & \text { Temperature of nanofluid }(\mathrm{K}) \\ T_{w} & \text { Hot fluid temperature at surface }(\mathrm{K}) \\ T_{\infty} & \text { Ambient fluid temperature }(\mathrm{K}) \\ U_{\infty} & \text { Velocity of ambient fluid }\left(\mathrm{m} \mathrm{s}^{-1}\right) \\ u & \text { Velocity component parallel to the } x \text {-axis }\left(\mathrm{m} \mathrm{s}^{-1}\right)\end{array}$

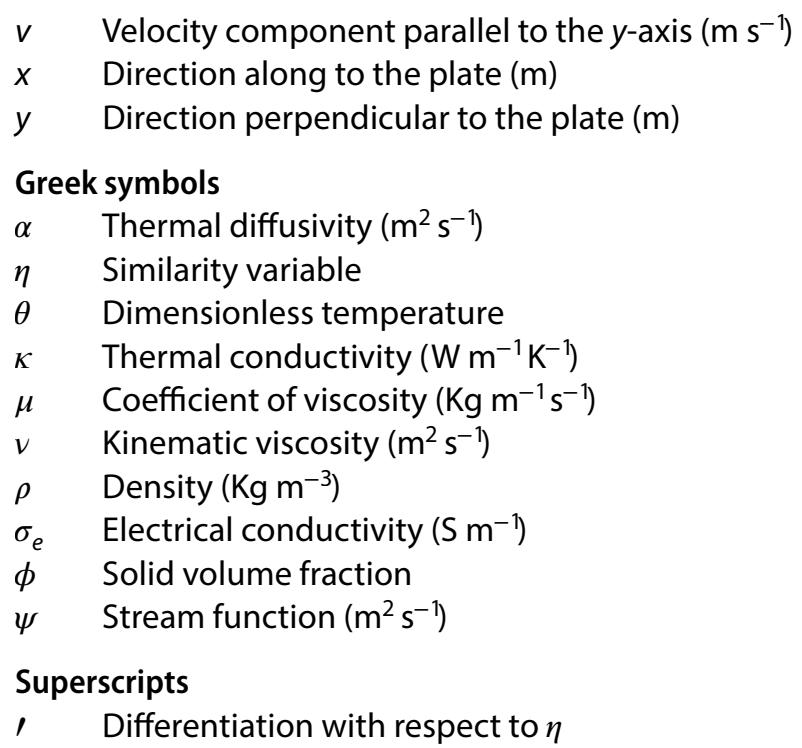

Santosh Chaudhary, d11.santosh@yahoo.com | 'Department of Mathematics, Malaviya National Institute of Technology, Jaipur 302017, India. 


\section{Subscripts \\ $f \quad$ Base fluid \\ nf Nanofluid \\ $s \quad$ Nano solid particles}

\section{Introduction}

Along the shear forces action, an effort done through the fluid on adjoining layers is converted into a heat, which is known as viscous dissipation. For higher velocity and viscous flows, the viscous dissipation impact on heat transfer is essential. Viscous dissipation appears in natural convection for different devices and also in powerful gravitational fields. Whereas, a process in which electric current energy is transformed into heat as it flows by resistance is called Joule heating or ohmic heating. Joule heating has a lot of applications in the area of technology and industrial processing. Some examples of applications are electric heaters and fuses, handling of food, electric stoves, electronic cigarette and incandescent light bulb. Initially, El-Amin [1] developed the combined influence of viscous dissipation and Joule heating and observed the convectional boundary layer flow situation towards the embedded porous medium. Moreover, numerous authors like as Abo-Eldahab and El-Aziz [2], Jat and Chaudhary [3], Yavari et al. [4] and Das et al. [5] have studied the fluid flow with viscous dissipation and Joule heating influences for some extended effects. In recent years, various explorations have been found by Hayat et al. [6], Hussain et al. [7] and Chaudhary and Choudhary [8] and analyzed the boundary layer flow in the presence of viscous dissipation and Joule heating.

To adjust the structure of boundary layer, an efficient technique as magnetohydrodynamic (MHD) principle has been used. MHD is an observation of electrically conducting fluids in a magnetic field, which depends on the induced magnetic field strength. Some examples of magneto fluids are liquid of metals, salt water, plasmas and electrolytes, and few applications of MHD effect such as boundary layer control in aerodynamics, geothermal energy, bearing, MHD generators and sensors, crystal growth, pumps, electromagnetic castings and plasma studies have significant scope in the areas of engineering and technologies. Alfven [9] pioneered the analysis of MHD field and analyzed the electromagnetic hydrodynamic waves existence. After that Ganesan and Palani [10] executed the study of unsteady MHD flow towards an inclined plate with natural convection. Moreover, Jat and Chaudhary [11], Butt and Ali [12], Imtiaz et al. [13], Chaudhary and Choudhary [14], Aydin and Selvitopi [15], Rehman et al. [16, 17] and Jha and Malgwi [18] have inscribed some articles on heat and mass transfer in MHD flow against to various situations.
The conventional ordinary fluids specifically oil, toluene, water and ethylene glycol have lower thermal conductivity and heat transfer rate capability. But increasing demand of advanced technology in electronic devices miniaturization have requirement of heat transfer medium behaving like a liquid, which has greater heat transfer efficiency to develop the thermal characteristics. This type of medium is known as nanofluid. Nanofluids have two types of materials namely base fluid and ultrafine nanoparticles with the diameter size 1-100 $\mathrm{nm}$. Some frequent nanoparticles made by metallic are silicon, titanium, copper, aluminum and silver. Nanofluids have drawn researchers awareness in the fields of engineering technology and science as a wide range of industrial applications such as food, drinks, dyes and toners, airplane engine, bio-chemical dispensation, microchip technology, pharmacological, dynamism, aerospace, remedial apparatus and devices. Choi [19] essentially discussed the concept of nanofluids for the enlargement of the thermal conductivity of fluids. Further, Buoyancy-driven heat transfer increment in a twodimensional enclosure by using nanofluids established by Khanafer et al. [20]. Latterly, Chein and Chuang [21], Yang and Lai [22], Mital [23], Ibrahim and Makinde [24], Makinde et al. [25], Chaudhary and Kanika [26], Rehman et al. [27], Kandasamy et al. [28], Ma et al. [29] and Sheikholeslami et al. [30] have inspected the numerous numerical and analytical explorations for the improvement of nanofluids heat transfer.

Newtonian heating is a way of heat transfer into the conventional fluid from the boundary surface with specific heat capacity. That type of composition appears in the setup of convection flows and heat introduces through solar radiation. The Newtonian heating impact is found if heat flux from the wall is proportional to the local surface temperature. This heating process utilized some practical base applications, particularly thermal energy storage, conjugate heat transport around fines, petroleum industry, nuclear turbines and heat exchanger. Merkin [31] illustrated the first analysis in the area of Newtonian heating and applied the effect of Newtonian heating on natural convection boundary layer flow via a vertical plate. Until, Lesnic et al. [32] established the free convection boundarylayer flow above a nearly horizontal surface in a porous medium with Newtonian heating. Further, an extensive literature on the behavior of Newtonian heating on boundary layer flow have been discussed by Makinde [33], Akbar and Khan [34], Hayat et al. [35], Chaudhary et al. [36] and Kamran and Wiwatanapataphee [37].

Keller-Box method is a very versatile modeling scheme. It is comparatively easy to understand and implemented. The Keller-Box technique works well for the two-dimensional model with simplified system geometries. This method is used directly to the differential form of the governing 
equations. The details of Keller-Box method can be found in the book by Vajravelu and Prasad [38].

Above mentioned literature pointed out that Newtonian heating effect on MHD boundary layer flow of electrically conducting fluid as water containing nanoparticles specifically silver $(\mathrm{Ag})$, copper $(\mathrm{Cu})$, titanium dioxide $\left(\mathrm{TiO}_{2}\right)$ and alumina $\left(\mathrm{Al}_{2} \mathrm{O}_{3}\right)$ is not examined yet. So the main objective of the present study is to extend the investigation of Makinde [39] with the impacts of viscous dissipation and Joule heating. Subsequently, the behaviors of nanofluids and considering parameters on the velocity, temperature, local skin friction coefficient and the local Nusselt number are given by plotted or tabulated values and discussed in detail.

\section{Flow analysis}

Consider an analysis of two-dimensional, steady boundary layer flow of viscous, electrically conducting nanofluids over a flat surface with Newtonian heating. It is assumed that there are thermal equilibrium and no-slip condition between base fluid water and nanoparticles like $\mathrm{Ag}, \mathrm{Cu}, \mathrm{TiO}_{2}$ and $\mathrm{Al}_{2} \mathrm{O}_{3}$. A coordinate system $(x, y)$ is chosen such that $x$ and $y$-directions are along to the plate and perpendicular to it respectively, and the flow is taken place at $y \geqslant 0$ as shown in Fig. 1. The hot fluid temperature $T_{w}$ at the surface is surmised, while the velocity and temperature of the ambient nanofluid are taken constant value $U_{\infty}$ and $T_{\infty}$ respectively. A uniform magnetic field of strength $B_{0}$ is applied parallel to the $y$-axis. For small value of the magnetic Reynolds number, the impact of an induced magnetic field is considered negligible. After that, the influences of viscous dissipation and Joule heating are considered. Under these assumptions, the basic governing equations are defined as (Bansal [40])

$\frac{\partial u}{\partial x}+\frac{\partial v}{\partial y}=0$

$u \frac{\partial u}{\partial x}+v \frac{\partial u}{\partial y}=v_{n f} \frac{\partial^{2} u}{\partial y^{2}}-\frac{\left(\sigma_{e}\right)_{n f} B_{0}^{2}}{\rho_{n f}}\left(u-U_{\infty}\right)$

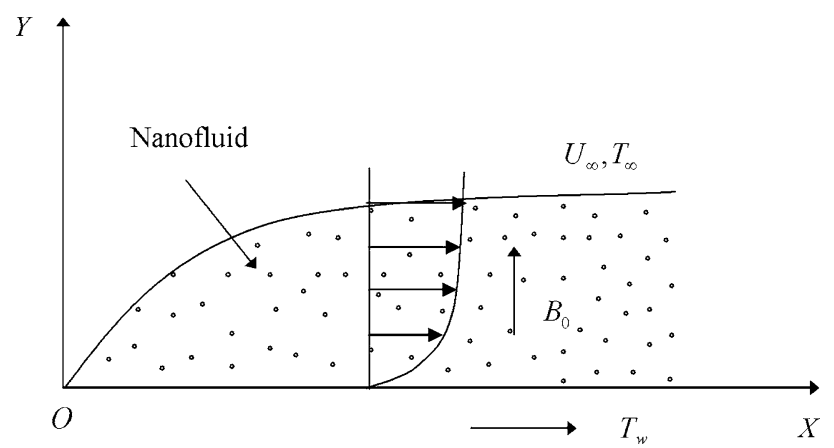

Fig. 1 Flow configuration

$$
\begin{aligned}
u \frac{\partial T}{\partial x}+v \frac{\partial T}{\partial y}= & \alpha_{n f} \frac{\partial^{2} T}{\partial y^{2}}+\frac{\mu_{n f}}{\left(\rho C_{p}\right)_{n f}}\left(\frac{\partial u}{\partial y}\right)^{2} \\
& +\frac{\left(\sigma_{e}\right)_{n f} B_{0}{ }^{2}}{\left(\rho C_{p}\right)_{n f}}\left(u-U_{\infty}\right)^{2}
\end{aligned}
$$

with the corresponding boundary conditions

$y=0: \quad u=0, \quad v=0, \quad-\kappa_{f} \frac{\partial T}{\partial y}=h_{t}\left(T_{w}-T\right)$

$y \rightarrow \infty: \quad u \rightarrow U_{\infty}, \quad T \rightarrow T_{\infty}$

where subscripts $n f$ and $f$ indicate the thermophysical properties of the nanofluid and the base fluid respectively, $u$ and $v$ are the velocity components parallel to the $x$ - and $y$-axes respectively, $\nu=\frac{\mu}{\rho}$ is the kinematic viscosity, $\mu$ is the coefficient of viscosity, $\rho$ is the density, $\sigma_{e}$ is the electrical conductivity, $T$ is the temperature of nanofluid, $\alpha=\frac{\kappa}{\rho C_{p}}$ is the thermal diffusivity, $\kappa$ is the thermal conductivity, $C_{p}$ is the specific heat at constant pressure and $h_{t}$ is the heat transfer coefficient.

Further, the nanofluid's thermophysical properties namely coefficient of viscosity, density, electrical conductivity, thermal conductivity and heat capacitance proceed from Mohyud-Din et al. [41] are given by

$\frac{\mu_{n f}}{\mu_{f}}=\frac{1}{(1-\phi)^{5 / 2}}$

$\frac{\rho_{n f}}{\rho_{f}}=1-\phi+\phi \frac{\rho_{s}}{\rho_{f}}$

$\frac{\left(\sigma_{e}\right)_{n f}}{\left(\sigma_{e}\right)_{f}}=\frac{2\left(\sigma_{e}\right)_{f}+\left(\sigma_{e}\right)_{s}-2 \phi\left[\left(\sigma_{e}\right)_{f}-\left(\sigma_{e}\right)_{s}\right]}{2\left(\sigma_{e}\right)_{f}+\left(\sigma_{e}\right)_{s}+\phi\left[\left(\sigma_{e}\right)_{f}-\left(\sigma_{e}\right)_{s}\right]}$

$\frac{\kappa_{n f}}{\kappa_{f}}=\frac{2 \kappa_{f}+\kappa_{s}-2 \phi\left(\kappa_{f}-\kappa_{s}\right)}{2 \kappa_{f}+\kappa_{s}+\phi\left(\kappa_{f}-\kappa_{s}\right)}$

$\frac{\left(\rho C_{p}\right)_{n f}}{\left(\rho C_{p}\right)_{f}}=1-\phi+\phi \frac{\left(\rho C_{p}\right)_{s}}{\left(\rho C_{p}\right)_{f}}$

where subscript $s$ represents the physical characteristics for nano solid particles and $\phi$ is the solid volume fraction. Moreover, Table 1 (Su and Zheng [42]) given the values for the above mentioned physical properties of conventional fluid and nanoparticles.

\section{Transformed problem}

Similarity variables are imported by Makinde [39] as follows 
Table 1 Thermophysical resources of water and nanoparticles

\begin{tabular}{llllll}
\hline Properties & Water & $\mathrm{Ag}$ & $\mathrm{Cu}$ & $\mathrm{TiO}_{2}$ & $\mathrm{Al}_{2} \mathrm{O}_{3}$ \\
\hline$\kappa\left(\mathrm{W} \mathrm{m}^{-1} \mathrm{~K}^{-1}\right)$ & 0.613 & 429 & 400 & 8.9538 & 40 \\
$\rho\left(\mathrm{Kg} \mathrm{m}^{-3}\right)$ & 997.1 & 10500 & 8933 & 4250 & 3970 \\
$C_{p}\left(\mathrm{~J} \mathrm{Kg}^{-1} \mathrm{~K}^{-1}\right)$ & 4179 & 235 & 385 & 686.2 & 765 \\
$\sigma_{e}\left(\mathrm{~S} \mathrm{~m}^{-1}\right)$ & 0.05 & $6.3 \times 10^{7}$ & $5.96 \times 10^{7}$ & $0.24 \times 10^{7}$ & $3.69 \times 10^{7}$ \\
\hline
\end{tabular}

$\psi=\left(v_{f} U_{\infty} x\right)^{1 / 2} f(\eta), \quad \eta=\left(\frac{U_{\infty}}{v_{f} x}\right)^{1 / 2} y$,

$T=T_{\infty}+\left(T_{w}-T_{\infty}\right) \theta(\eta)$

where $\psi(x, y)$ is the stream function, which is utilized in the usual manner as $u=\frac{\partial \psi}{\partial y}$ and $v=-\frac{\partial \psi}{\partial x}$ and symmetrically satisfied the continuity Eq. (1), $f(\eta)$ is the dimensionless stream function, $\eta$ is the similarity variable and $\theta(\eta)$ is the dimensionless temperature.

Using the similarity variables Eq. (10), the boundary layer Eqs. (2) and (3) with the associated boundary conditions Eq. (4) can be replaced as

$$
\begin{aligned}
& f^{\prime \prime \prime}+\frac{1}{2}(1-\phi)^{5 / 2}\left(1-\phi+\phi \frac{\rho_{s}}{\rho_{f}}\right) f f^{\prime \prime} \\
&-\frac{\left(\sigma_{e}\right)_{n f}}{\left(\sigma_{e}\right)_{f}}(1-\phi)^{5 / 2} M\left(f^{\prime}-1\right)=0 \\
& \frac{\kappa_{n f}}{\kappa_{f}} \theta^{\prime \prime}+\frac{1}{2}\left[1-\phi+\phi \frac{\left(\rho C_{p}\right)_{s}}{\left(\rho C_{p}\right)_{f}}\right] \operatorname{Prf} \theta^{\prime} \\
&+\operatorname{Br}\left[\frac{1}{(1-\phi)^{5 / 2}} f^{\prime \prime^{2}}+\frac{\left(\sigma_{e}\right)_{n f}}{\left(\sigma_{e}\right)_{f}} M\left(f^{\prime}-1\right)^{2}\right]=0
\end{aligned}
$$

subject to the relevant boundary conditions

$$
\eta=0: f=0, \quad f^{\prime}=0, \quad \theta^{\prime}=\operatorname{Bi}(\theta-1)
$$$$
\eta \rightarrow \infty: f^{\prime} \rightarrow 1, \quad \theta \rightarrow 0
$$

where prime ( $($ ) denotes the differentiation with respect to $\eta, M=\frac{\left(\sigma_{e}\right)_{f} B_{0}{ }^{2} v_{f} R e_{x}}{\rho_{f} U_{\infty}{ }^{2}}$ is the magnetic parameter, $R e_{x}=\frac{U_{\infty} x}{v_{f}}$ is the local Reynolds number, $\operatorname{Pr}=\frac{v_{f}}{\alpha_{f}}$ is the Prandtl number, $B r=\frac{\mu_{f} U_{\infty}{ }^{2}}{\kappa_{f}\left(T_{\mu}-T_{\infty}\right)}$ is the Brinkmann number and $B i=\frac{h_{t} v_{f}}{\kappa_{f} U_{\infty}} \operatorname{Re}_{x}^{\kappa_{f}(1 / 2)}$ is the local Biot number.

\section{Declaration of curiosity}

The local skin friction coefficient $C_{f}$ and the local Nusselt number $N u_{x}$ are the physical quantities of primary interest, which are expressed as
$C_{f}=\frac{\mu_{n f}\left(\frac{\partial u}{\partial y}\right)_{y=0}}{\frac{\rho_{f} U_{\infty}^{2}}{2}}, \quad N u_{x}=-\frac{\kappa_{n f} X\left(\frac{\partial T}{\partial y}\right)_{y=0}}{\kappa_{f}\left(T_{w}-T_{\infty}\right)}$

By using the non-dimensional variables Eq. (10), the physical quantities Eq. (14) can be defined as

$R e_{x}{ }^{1 / 2} C_{f}=\frac{2}{(1-\phi)^{5 / 2}} f^{\prime \prime}(0), \quad \frac{1}{R e_{x}^{1 / 2}} N u_{x}=-\frac{\kappa_{n f}}{\kappa_{f}} \theta^{\prime}(0)$

\section{Solution methodology}

The system of the nonlinear ordinary differential Eqs. (11) and (12) along with the associated boundary conditions Eq. (13) is solved numerically with a finite difference scheme as Keller-Box method (Kumar and Sood [43]). For the computational procedure, the suitable finite value of the far field boundary condition as $\eta \rightarrow \infty=6$ is assumed.

\subsection{Scheme of implicit finite difference}

Introducing

$f^{\prime}=p$

$p^{\prime}=q$

$\theta^{\prime}=s$

so the Eqs. (11) and (12) can be given as

$$
\begin{aligned}
q^{\prime} & +\frac{1}{2}(1-\phi)^{5 / 2}\left(1-\phi+\phi \frac{\rho_{s}}{\rho_{f}}\right) f q \\
- & \frac{\left(\sigma_{e}\right)_{n f}}{\left(\sigma_{e}\right)_{f}}(1-\phi)^{5 / 2} M(p-1)=0
\end{aligned}
$$

$$
\begin{aligned}
& \frac{\kappa_{n f}}{\kappa_{f}} s^{\prime}+\frac{1}{2}\left[1-\phi+\phi \frac{\left(\rho C_{p}\right)_{s}}{\left(\rho C_{p}\right)_{f}}\right] \text { Prfs } \\
& \quad+B r\left[\frac{1}{(1-\phi)^{5 / 2}} q^{2}+\frac{\left(\sigma_{e}\right)_{n f}}{\left(\sigma_{e}\right)_{f}} M(p-1)^{2}\right]=0
\end{aligned}
$$

along with the corresponding boundary conditions Eq. (13) become as follows

$\eta=0: f=0, \quad p=0, \quad s=\operatorname{Bi}(\theta-1)$

$\eta=6: p \rightarrow 1, \quad \theta \rightarrow 0$

The rectangular grid $X \eta$-plane and net points are defined by Fig. 2 , such as 


$$
\begin{aligned}
& x_{0}=0, x_{i}=x_{i-1}+k_{i}, i=1,2,3, \ldots l \\
& \eta_{0}=0, \eta_{j}=\eta_{j-1}+h_{j}, j=1,2,3, \ldots J
\end{aligned}
$$

By using centered difference derivatives, the finite difference form of the Eqs. (16) to (20) for the mid point $\left(x_{i}, \eta_{j-\frac{1}{2}}\right)$ of the segment $Q R$ are defined as

$$
\begin{aligned}
& f_{j}-f_{j-1}-\frac{1}{2} h_{j}\left(p_{j}+p_{j-1}\right)=0 \\
& p_{j}-p_{j-1}-\frac{1}{2} h_{j}\left(q_{j}+q_{j-1}\right)=0 \\
& \theta_{j}-\theta_{j-1}-\frac{1}{2} h_{j}\left(s_{j}+s_{j-1}\right)=0 \\
& q_{j}-q_{j-1}+\frac{1}{8} h_{j}(1-\phi)^{5 / 2}\left(1-\phi+\phi \frac{\rho_{s}}{\rho_{f}}\right) \\
& \quad \times\left(f_{j}+f_{j-1}\right)\left(q_{j}+q_{j-1}\right) \\
& \quad-\frac{1}{2} h_{j} \frac{\left(\sigma_{e}\right)_{n f}}{\left(\sigma_{e}\right)_{f}}(1-\phi)^{5 / 2} M\left(p_{j}+p_{j-1}-1\right)=0 \\
& \frac{\kappa_{n f}}{\kappa_{f}}\left(s_{j}-s_{j-1}\right)+\frac{1}{8} h_{j}\left[1-\phi+\phi \frac{\left(\rho C_{p}\right)_{s}}{\left(\rho C_{p}\right)_{f}}\right] \\
& \quad \times \operatorname{Pr}\left(f_{j}+f_{j-1}\right)\left(s_{j}+s_{j-1}\right) \\
& \quad+\frac{1}{4} h_{j} B r\left[\frac{1}{(1-\phi)^{5 / 2}}\left(q_{j}+q_{j-1}\right)^{2}\right. \\
& \left.\quad+\frac{\left(\sigma_{e}\right)_{n f}}{\left(\sigma_{e}\right)_{f}} M\left(p_{j}+p_{j-1}-1\right)^{2}\right]=0
\end{aligned}
$$

Equations (23) to (27) are exhibited for $j=1,2,3, \ldots J-1$ and from the appropriate boundary conditions Eq. (21) it is prominent that

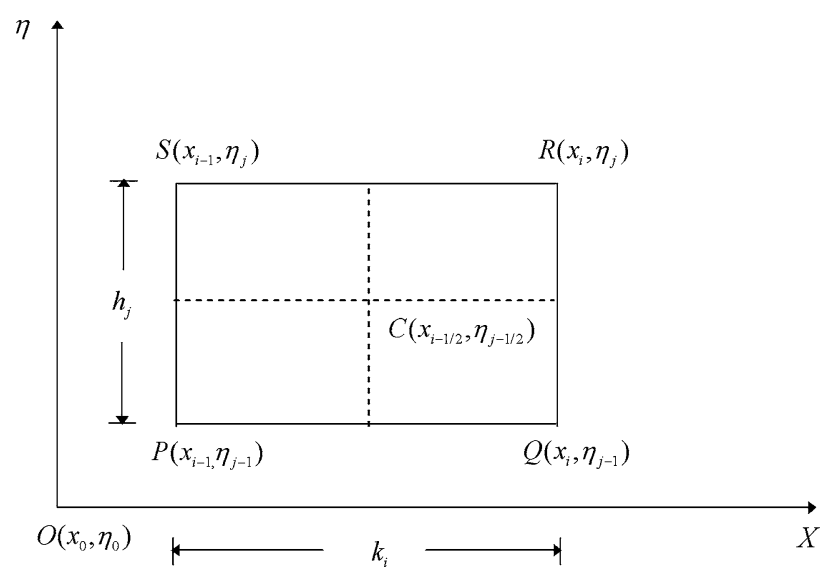

Fig. 2 Domain schematic representation $f_{0}=0, p_{0}=0, s_{0}=B i\left(\theta_{0}-1\right), p_{J} \rightarrow 1, \theta_{J} \rightarrow 0$

\subsection{Newton's method}

A nonlinear system is converted into a linear system by applying the Newton's method. So the following iterations are established

$f_{j}^{(i+1)}=f_{j}^{(i)}+\delta f_{j}^{(i)}, p_{j}^{(i+1)}=p_{j}^{(i)}+\delta p_{j}^{(i)}$,

$q_{j}^{(i+1)}=q_{j}^{(i)}+\delta q_{j}^{(i)}$,

$\theta_{j}^{(i+1)}=\theta_{j}^{(i)}+\delta \theta_{j}^{(i)}, s_{j}^{(i+1)}=s_{j}^{(i)}+\delta s_{j}^{(i)}$

Substituting the above considered expressions into the Eqs. (23) to (27) and then neglect the quadratic and higher order terms in $\delta f_{j}^{(i)}, \delta p_{j}^{(i)}, \delta q_{j}^{(i)}, \delta \theta_{j}^{(i)}$ and $\delta s_{j}^{(i)}$. Thus above scheme yields a tridiagonal model as follows

$$
\begin{aligned}
& \delta f_{j}-\delta f_{j-1}-\frac{1}{2} h_{j}\left(\delta p_{j}+\delta p_{j-1}\right)=\left(r_{1}\right)_{j-\frac{1}{2}} \\
& \delta p_{j}-\delta p_{j-1}-\frac{1}{2} h_{j}\left(\delta q_{j}+\delta q_{j-1}\right)=\left(r_{2}\right)_{j-\frac{1}{2}} \\
& \delta \theta_{j}-\delta \theta_{j-1}-\frac{1}{2} h_{j}\left(\delta s_{j}+\delta s_{j-1}\right)=\left(r_{3}\right)_{j-\frac{1}{2}} \\
& \left(a_{1}\right)_{j-\frac{1}{2}} \delta f_{j}+\left(a_{2}\right)_{j-\frac{1}{2}} \delta f_{j-1}+\left(a_{3}\right)_{j-\frac{1}{2}} \delta p_{j} \\
& \quad+\left(a_{4}\right)_{j-\frac{1}{2}} \delta p_{j-1}+\left(a_{5}\right)_{j-\frac{1}{2}} \delta q_{j}+\left(a_{6}\right)_{j-\frac{1}{2}} \delta q_{j-1}=\left(r_{4}\right)_{j-\frac{1}{2}} \\
& \left(b_{1}\right)_{j-\frac{1}{2}} \delta f_{j}+\left(b_{2}\right)_{j-\frac{1}{2}} \delta f_{j-1}+\left(b_{3}\right)_{j-\frac{1}{2}} \delta p_{j} \\
& \quad+\left(b_{4}\right)_{j-\frac{1}{2}} \delta p_{j-1}+\left(b_{5}\right)_{j-\frac{1}{2}} \delta q_{j}+\left(b_{6}\right)_{j-\frac{1}{2}} \delta q_{j-1} \\
& \quad+\left(b_{7}\right)_{j-\frac{1}{2}} \delta s_{j}+\left(b_{8}\right)_{j-\frac{1}{2}} \delta s_{j-1}=\left(r_{5}\right)_{j-\frac{1}{2}}
\end{aligned}
$$

where 


$$
\begin{aligned}
& \left(a_{1}\right)_{j-\frac{1}{2}}=\left(a_{2}\right)_{j-\frac{1}{2}}=\frac{1}{8} h_{j}(1-\phi)^{5 / 2}\left(1-\phi+\phi \frac{\rho_{s}}{\rho_{f}}\right)\left(q_{j}+q_{j-1}\right) \text {, } \\
& \left(a_{3}\right)_{j-\frac{1}{2}}=\left(a_{4}\right)_{j-\frac{1}{2}}=-\frac{1}{2} h_{j} \frac{\left(\sigma_{e}\right)_{n f}}{\left(\sigma_{e}\right)_{f}}(1-\phi)^{5 / 2} M \text {, } \\
& \left(a_{5}\right)_{j-\frac{1}{2}}=1+\frac{1}{8} h_{j}(1-\phi)^{5 / 2}\left(1-\phi+\phi \frac{\rho_{s}}{\rho_{f}}\right)\left(f_{j}+f_{j-1}\right) \text {, } \\
& \left(a_{6}\right)_{j-\frac{1}{2}}=-1+\frac{1}{8} h_{j}(1-\phi)^{5 / 2}\left(1-\phi+\phi \frac{\rho_{s}}{\rho_{f}}\right)\left(f_{j}+f_{j-1}\right), \\
& \left(b_{1}\right)_{j-\frac{1}{2}}=\left(b_{2}\right)_{j-\frac{1}{2}}=\frac{1}{8} h_{j}\left[1-\phi+\phi \frac{\left(\rho C_{p}\right)_{s}}{\left(\rho C_{p}\right)_{f}}\right] \operatorname{Pr}\left(s_{j}+s_{j-1}\right), \\
& \left(b_{3}\right)_{j-\frac{1}{2}}=\left(b_{4}\right)_{j-\frac{1}{2}}=\frac{1}{4} h_{j} \frac{\left(\sigma_{e}\right)_{n f}}{\left(\sigma_{e}\right)_{f}} \operatorname{MBr}\left(p_{j}+p_{j-1}-2\right) \text {, } \\
& \left(b_{5}\right)_{j-\frac{1}{2}}=\left(b_{6}\right)_{j-\frac{1}{2}}=\frac{1}{4} h_{j} \frac{1}{(1-\phi)^{5 / 2}} \operatorname{Br}\left(q_{j}+q_{j-1}\right) \text {, } \\
& \left(b_{7}\right)_{j-\frac{1}{2}}=\frac{\kappa_{n f}}{\kappa_{f}}+\frac{1}{8} h_{j}\left[1-\phi+\phi \frac{\left(\rho C_{p}\right)_{s}}{\left(\rho C_{p}\right)_{f}}\right] \operatorname{Pr}\left(f_{j}+f_{j-1}\right), \\
& \left(b_{8}\right)_{j-\frac{1}{2}}=-\frac{\kappa_{n f}}{\kappa_{f}}+\frac{1}{8} h_{j}\left[1-\phi+\phi \frac{\left(\rho C_{p}\right)_{s}}{\left(\rho C_{p}\right)_{f}}\right] \operatorname{Pr}\left(f_{j}+f_{j-1}\right) \text {, } \\
& \left(r_{1}\right)_{j-\frac{1}{2}}=-f_{j}+f_{j-1}+\frac{1}{2} h_{j}\left(p_{j}+p_{j-1}\right) \text {, } \\
& \left(r_{2}\right)_{j-\frac{1}{2}}=-p_{j}+p_{j-1}+\frac{1}{2} h_{j}\left(q_{j}+q_{j-1}\right) \text {, } \\
& \left(r_{3}\right)_{j-\frac{1}{2}}=-\theta_{j}+\theta_{j-1}+\frac{1}{2} h_{j}\left(s_{j}+s_{j-1}\right) \text {, } \\
& \left(r_{4}\right)_{j-\frac{1}{2}}=-\left(q_{j}-q_{j-1}\right)-\frac{1}{8} h_{j}(1-\phi)^{5 / 2}\left(1-\phi+\phi \frac{\rho_{s}}{\rho_{f}}\right) \\
& \times\left(f_{j}+f_{j-1}\right)\left(q_{j}+q_{j-1}\right) \\
& +\frac{1}{2} h_{j} \frac{\left(\sigma_{e}\right)_{n f}}{\left(\sigma_{e}\right)_{f}}(1-\phi)^{5 / 2} M\left(p_{j}+p_{j-1}-1\right)
\end{aligned}
$$

and

$$
\begin{aligned}
\left(r_{5}\right)_{j-\frac{1}{2}}= & -\frac{\kappa_{n f}}{\kappa_{f}}\left(s_{j}-s_{j-1}\right) \\
& -\frac{1}{8} h_{j}\left[1-\phi+\phi \frac{\left(\rho C_{p}\right)_{s}}{\left(\rho C_{p}\right)_{f}}\right] \\
& \times \operatorname{Pr}\left(f_{j}+f_{j-1}\right)\left(s_{j}+s_{j-1}\right) \\
& -\frac{1}{4} h_{j} B r\left[\frac{1}{(1-\phi)^{5 / 2}}\left(q_{j}+q_{j-1}\right)^{2}\right. \\
& \left.+\frac{\left(\sigma_{e}\right)_{n f}}{\left(\sigma_{e}\right)_{f}} M\left(p_{j}+p_{j-1}-1\right)^{2}\right]
\end{aligned}
$$

For all iterations, it is taken as

$\delta f_{0}=0, \delta p_{0}=0, \delta s_{0}=0, \delta p_{\jmath}=0, \delta \theta_{\jmath}=0$

\subsection{Method of block elimination}

Block tridiagonal matrix form of Eqs. (30) to (34) is
$\left[\begin{array}{ccccccc}{\left[A_{1}\right]} & {\left[C_{1}\right]} & & & & & \\ {\left[B_{2}\right]} & {\left[A_{2}\right]} & {\left[C_{2}\right]} & & & & \\ & & & \ddots & \ldots & & \\ & & & & {\left[B_{J-1}\right]} & {\left[A_{J-1}\right]} & {\left[C_{J-1}\right]} \\ & & & & & {\left[B_{J}\right]} & {\left[A_{J}\right]}\end{array}\right]\left[\begin{array}{c}{\left[\delta_{1}\right]} \\ {\left[\delta_{2}\right]} \\ \vdots \\ {\left[\delta_{J-1}\right]} \\ {\left[\delta_{J}\right]}\end{array}\right]$

$[A][\delta]=[r]$

where

$\begin{aligned} {\left[A_{1}\right] } & =\left[\begin{array}{lllll}0 & 0 & 1 & 0 & 0 \\ \frac{-h_{j}}{2} & 0 & 0 & \frac{-h_{j}}{2} & 0 \\ 0 & -1 & 0 & 0 & \frac{-h_{j}}{2} \\ a_{6} & 0 & a_{1} & a_{5} & 0 \\ b_{6} & 0 & b_{1} & b_{5} & b_{7}\end{array}\right] ; \\ {\left[A_{j}\right] } & =\left[\begin{array}{lllll}\frac{-h_{j}}{2} & 0 & 1 & 0 & 0 \\ -1 & 0 & 0 & \frac{-h_{j}}{2} & 0 \\ 0 & -1 & 0 & 0 & \frac{-h_{j}}{2} \\ a_{4} & 0 & a_{1} & a_{5} & 0 \\ b_{4} & 0 & b_{1} & b_{5} & b_{7}\end{array}\right], 2 \leq j \leq J ;\end{aligned}$

$\left[B_{j}\right]=\left[\begin{array}{ccccc}0 & 0 & -1 & 0 & 0 \\ 0 & 0 & 0 & \frac{-h_{j}}{2} & 0 \\ 0 & 0 & 0 & 0 & \frac{-h_{j}}{2} \\ 0 & 0 & a_{4} & a_{2} & 0 \\ 0 & 0 & b_{4} & b_{8} & b_{2}\end{array}\right], \quad 2 \leq j \leq J ;$

$\left[C_{j}\right]=\left[\begin{array}{lllll}\frac{-h_{j}}{2} & 0 & 0 & 0 & 0 \\ 1 & 0 & 0 & 0 & 0 \\ 0 & 1 & 0 & 0 & 0 \\ a_{3} & 0 & 0 & 0 & 0 \\ b_{3} & 0 & 0 & 0 & 0\end{array}\right], \quad 1 \leq j \leq J-1 ;$

$\left[\delta_{1}\right]=\left[\begin{array}{l}\delta q_{0} \\ \delta \theta_{0} \\ \delta f_{1} \\ \delta q_{1} \\ \delta s_{1}\end{array}\right] ; \quad\left[\delta_{j}\right]=\left[\begin{array}{l}\delta p_{j-1} \\ \delta \theta_{j-1} \\ \delta f_{j} \\ \delta q_{j} \\ \delta s_{j}\end{array}\right], \quad 2 \leq j \leq J$ and

$\left[r_{j}\right]=\left[\begin{array}{l}\left(r_{1}\right)_{j} \\ \left(r_{2}\right)_{j} \\ \left(r_{3}\right)_{j} \\ \left(r_{4}\right)_{j} \\ \left(r_{5}\right)_{j}\end{array}\right], \quad 1 \leq j \leq J$. 
Matrix $A$ is taken as a nonsingular matrix to solve the Eq. (36) then the matrix $A$ can be written in the product of the lower triangular matrix and the upper triangular matrix as

$[A]=[L][U]$

where

$$
\begin{gathered}
{[L]=\left[\begin{array}{lllll}
{\left[\alpha_{1}\right]} & & & & \\
{\left[B_{2}\right]} & {\left[\alpha_{2}\right]} & & & \\
& & \ddots & & \\
& & & {\left[\alpha_{J-1}\right]} & \\
& & & {\left[B_{J}\right]} & {\left[\alpha_{J}\right]}
\end{array}\right] \text { and }} \\
{[U]=\left[\begin{array}{ccccc}
I & {\left[\beta_{1}\right]} & & & \\
& l & {\left[\beta_{2}\right]} & & \\
& & \ddots & \ddots & \\
& & & l & {\left[\beta_{J-1}\right]} \\
& & & & l
\end{array}\right]}
\end{gathered}
$$

with $/$ is the identity matrix of order $5 \times 5$, and $\left[\alpha_{j}\right]$ and $\left[\beta_{j}\right]$ are the matrices of order $5 \times 5$, whose elements are found through the following equations

$\left[\alpha_{1}\right]=\left[A_{1}\right]$

$\left[A_{1}\right]\left[\beta_{1}\right]=\left[C_{1}\right]$

$\left[\alpha_{j}\right]=\left[A_{j}\right]-\left[B_{j}\right]\left[\beta_{j-1}\right], \quad j=2,3, \ldots, J$

In view of

$[L][U]\left[\delta_{j}\right]=\left[r_{j}\right]$

with the assumption as

$[U]\left[\delta_{j}\right]=\left[W_{j}\right]$
$[L]\left[W_{j}\right]=\left[r_{j}\right]$

where $\left[W_{j}\right]$ is the column matrix of order $5 \times 1$ and elements of $\left[W_{j}\right]$ can be discovered by Eq. (43) as

$\left[\alpha_{1}\right]\left[W_{1}\right]=\left[r_{1}\right]$

$\left[\alpha_{j}\right]\left[W_{j}\right]=\left[r_{j}\right]-\left[B_{j}\right]\left[W_{j-1}\right], \quad 2 \leq j \leq J$

the Eq. (42) disposes the solution, whose elements can be carried from the given relations

$\left[\delta_{j}\right]=\left[W_{j}\right]-\left[\beta_{j}\right]\left[\delta_{j+1}\right], \quad 1 \leq j \leq J-1$

$\left[\delta_{\jmath}\right]=\left[W_{\jmath}\right]$

These iterative processes are repeated until some convergence regulation is satisfied with maintaining accuracy of $10^{-7}$, while process is stopped when $\left|\delta q_{0}^{(i)}\right| \leq \xi$, where $\xi$ is a small prescribed value.

\section{Verification of the numerical method}

Table 2 depicts the comparison made with earlier values by Makinde [39], to validate the present computational results of wall shear stress $f^{\prime \prime}(0)$ for several values of the solid volume fraction $\phi$ along with the nanofluids included base fluid water and different nanoparticles namely $\mathrm{Cu}$, $\mathrm{TiO}_{2}$ and $\mathrm{Al}_{2} \mathrm{O}_{3}$ likewise in the absence of magnetic field. This table shows that numerical results are in good agreement with the previously published data, which validate the proposed method.
Table 2 Comparison of $f^{\prime \prime}(0)$ for various values of $\phi$ corresponding to different types of nanofluids with $M=0$

\begin{tabular}{llllllll}
\hline$\phi$ & \multicolumn{2}{l}{ Makinde [39] } & & & \multicolumn{3}{l}{ Present results } \\
& Cu-water & $\mathrm{TiO}_{2}$-water & $\mathrm{Al}_{2} \mathrm{O}_{3}$-water & & Cu-water & $\mathrm{TiO}_{2}$-water & $\mathrm{Al}_{2} \mathrm{O}_{3}$-water \\
\hline 0.000 & 0.3321 & 0.3321 & 0.3321 & & 0.33258 & 0.33258 & 0.33258 \\
0.008 & 0.3459 & 0.3398 & 0.3394 & & 0.33945 & 0.33350 & 0.33315 \\
0.014 & 0.3563 & 0.3456 & 0.3449 & & 0.34429 & 0.33410 & 0.33350 \\
0.016 & 0.3597 & 0.3476 & 0.3468 & & 0.34585 & 0.33430 & 0.33360 \\
0.020 & 0.3667 & 0.3515 & 0.3506 & & 0.34888 & 0.33466 & 0.33379 \\
0.100 & 0.5076 & 0.4362 & 0.4316 & & 0.39015 & 0.33569 & 0.33418 \\
\hline
\end{tabular}




\section{Discussion of physical outcomes}

To discuss the numerical values of the considered problem, the effects of the different types of nanofluids and the effects of the pertinent parameters namely the solid volume fraction $\phi$, the magnetic parameter $M$, the Brinkmann number $B r$ and the local Biot number $B i$ along with the Cu-water nanofluid on the velocity $f^{\prime}(\eta)$ and the temperature $\theta(\eta)$ distributions are plotted graphically. Subsequently, the computational values of the wall shear stress $f^{\prime \prime}(0)$ and the wall heat flux $\theta^{\prime}(0)$ for the impacts of the different nanofluids as well as the specified parameters are given via table. It is also noted that due to find the impact of anyone specified parameter, all remaining controlling parameters are taken constant.

Figures 3 and 4 display the influences of the four various types of nanofluids on the velocity $f^{\prime}(\eta)$ and the temperature $\theta(\eta)$ fields respectively. It can be seen from these figures that the fluid flow reduces and the fluid temperature enhances as the nanofluid changes in the
Fig. 3 Effect of different types of nanofluids on dimensionless velocity for $\phi=0.07$ and $M=0.01$

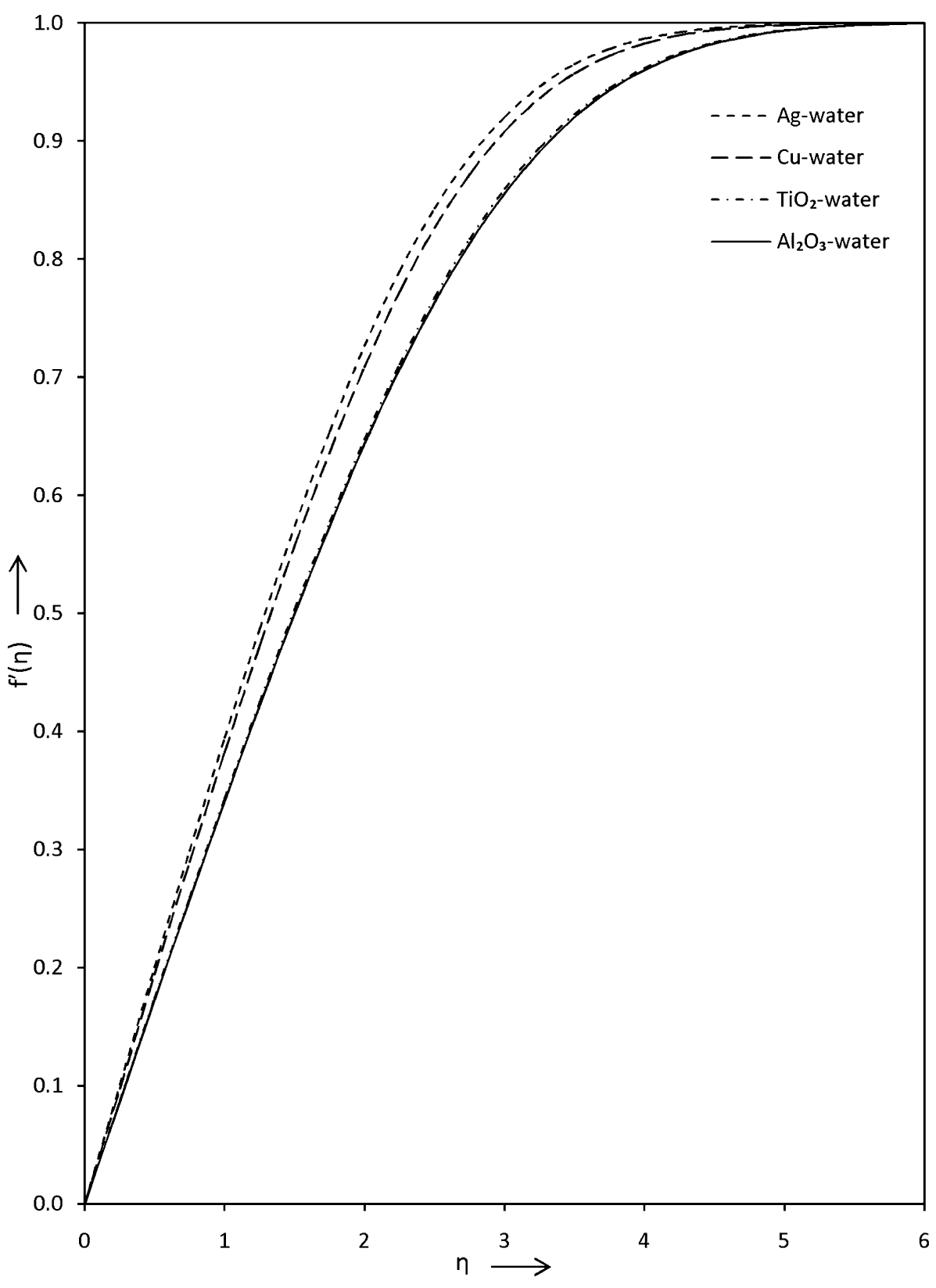


Fig. 4 Effect of different types of nanofluids on dimensionless temperature for $\phi=0.07$, $M=0.01, \operatorname{Pr}=6.2, B r=0.1$ and $B i=0.1$

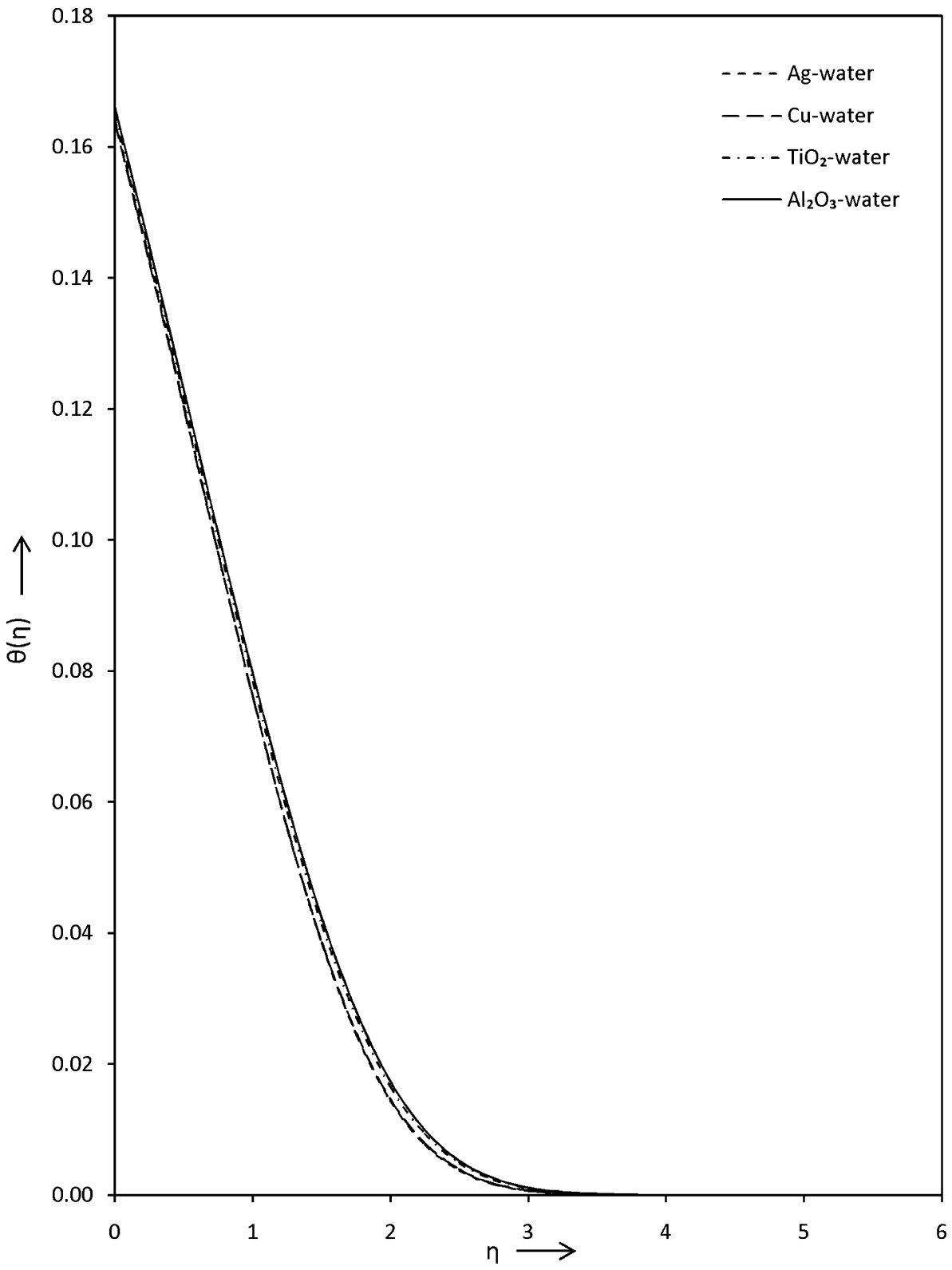

serial as $\mathrm{Ag}$-water, $\mathrm{Cu}$-water, $\mathrm{TiO}_{2}$-water and $\mathrm{Al}_{2} \mathrm{O}_{3}$-water respectively. This is true with the fact that the velocity and the temperature profiles modify according to the change of solid nanoparticles beside in base fluid water, because various types of nanoparticles have distinct mechanical and physical characteristics like as dynamic viscosity, density and thermal expansion coefficients.

Impacts on the dimensionless velocity $f^{\prime}(\eta)$ and the dimensionless temperature $\theta(\eta)$ for the several values of the solid volume fraction $\phi$ are presented by Figs. 5 and
6 respectively. These figures indicate that the velocity profile as well as the temperature profile rises with increment of the solid volume fraction $\phi$. Because, nanofluids have solid nanoparticles, which transport the more flow resistance. Further, enhancement of solid nanoparticles volume fraction leads to increment in the nanofluid thermal conductivity that in turn outcomes the enhancement in the temperature.

Figures 7 and 8 illustrate the influence of the magnetic parameter $M$ on the velocity $f^{\prime}(\eta)$ and the temperature $\theta(\eta)$ 
Fig. 5 Effect of $\phi$ with Cu-water nanofluid on dimensionless velocity for $M=0.01$

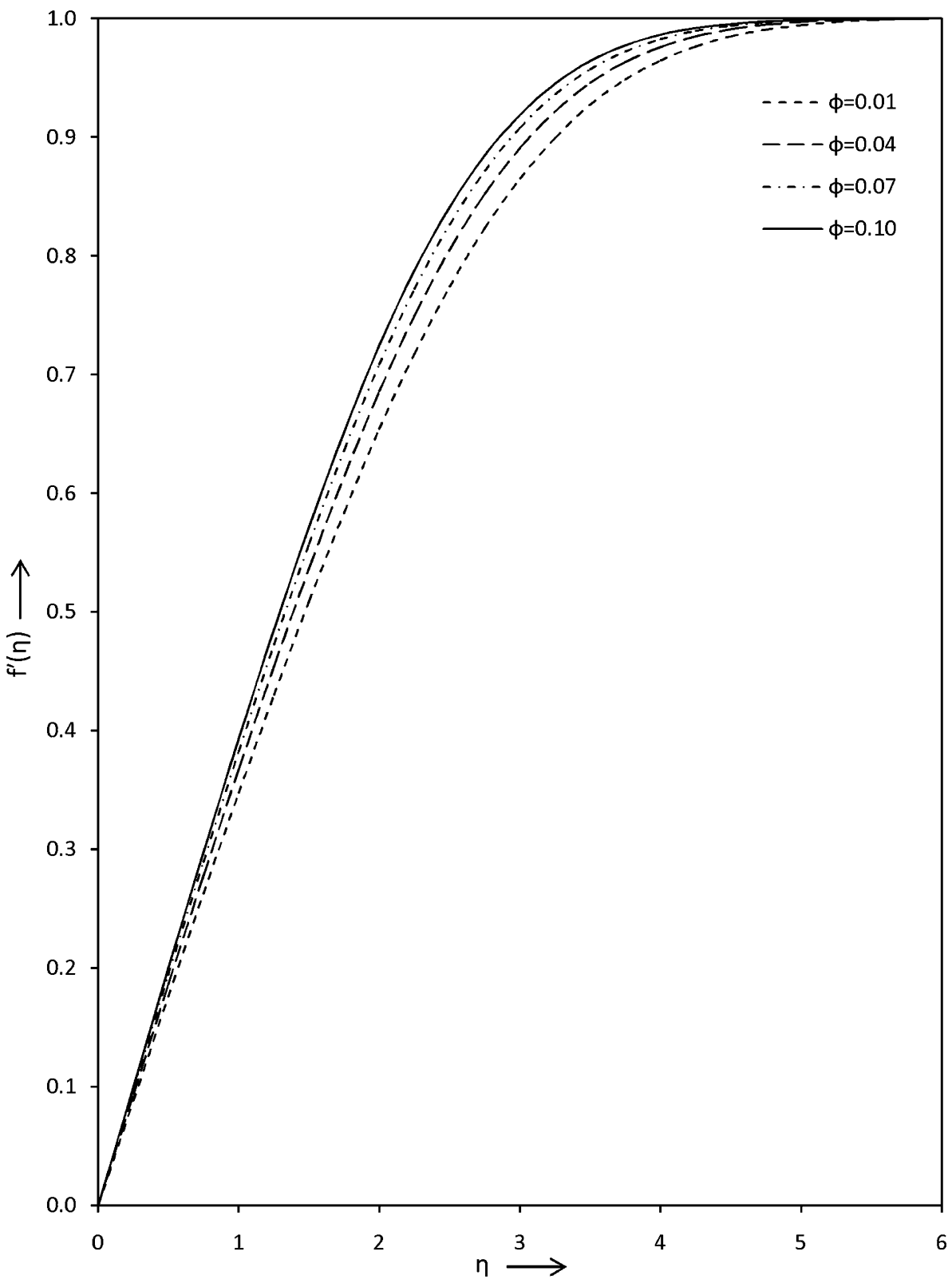

distributions respectively. It can be noted from these figures that the fluid velocity and the temperature increase along with the increment of the magnetic parameter $M$, while reverse is happened in temperature for $\eta>1$. Physically it occurs because ratio of electromagnetic force and the viscous force is equivalent to the magnetic parameter. So the Lorentz force rises with the increasing values of the magnetic parameter, which creates more resistance to the transport circumstance and opposes the fluid motion which composed heat.
Behavior of the Brinkmann number $\mathrm{Br}$ on the fluid temperature $\theta(\eta)$ is described in Fig. 9. This figure expresses that an enlargement in the Brinkmann number $B r$ leads to rise in temperature. This is occurred due to the reason that viscous dissipation impact on the flow area is to raise the energy, which accommodating the higher fluid temperature and buoyancy force. Increment in the buoyancy force along the developing dissipation parameter increases the temperature field. 
Fig. 6 Effect of $\phi$ with Cu-water nanofluid on dimensionless temperature for $M=0.01$,

$\operatorname{Pr}=6.2, B r=0.1$ and $B i=0.1$

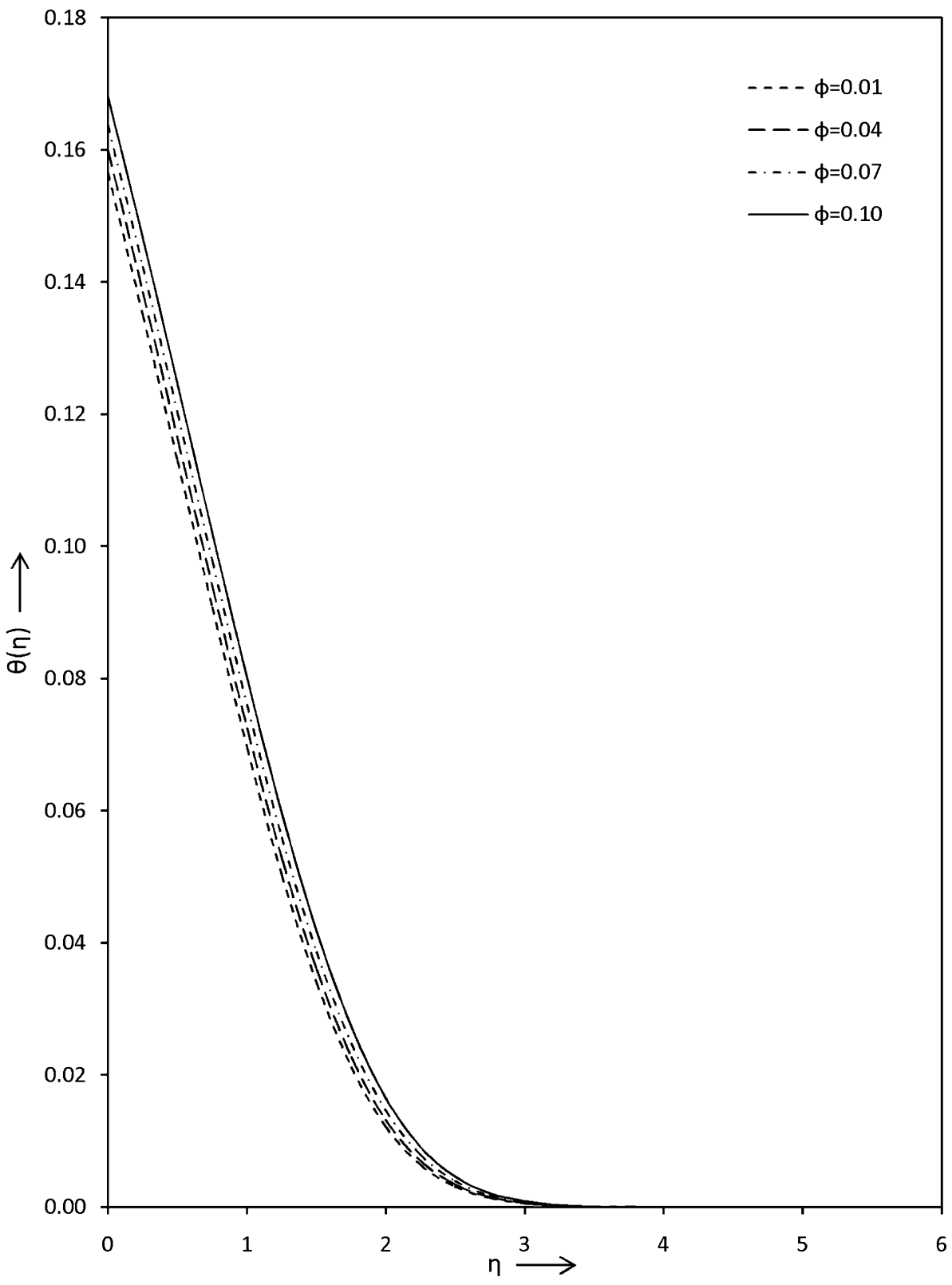

Figure 10 portrayed to indicate the temperature $\theta(\eta)$ profile for several values of the local Biot number $B i$. As the value of the local Biot number Bi step-up, temperature develops. From the physical point of view, surface thermal resistance reduces with the increasing nature of the local Biot number and so convective heat transfer to the fluid enhances.
Reflexion of the four different types of the nanofluids and several values of the solid volume fraction $\phi$, the magnetic parameter $M$, the Brinkmann number $\mathrm{Br}$ and the local Biot number $\mathrm{Bi}$ on the surface shear stress $f^{\prime \prime}(0)$ and the heat transfer rate $\theta^{\prime}(0)$ are exhibit through Table 3. It can be observed by Eq. (15) that $f^{\prime \prime}(0)$ and $\theta^{\prime}(0)$ are proportional to the local skin friction 
Fig. 7 Effect of $M$ with $C u$ water nanofluid on dimensionless velocity for $\phi=0.07$

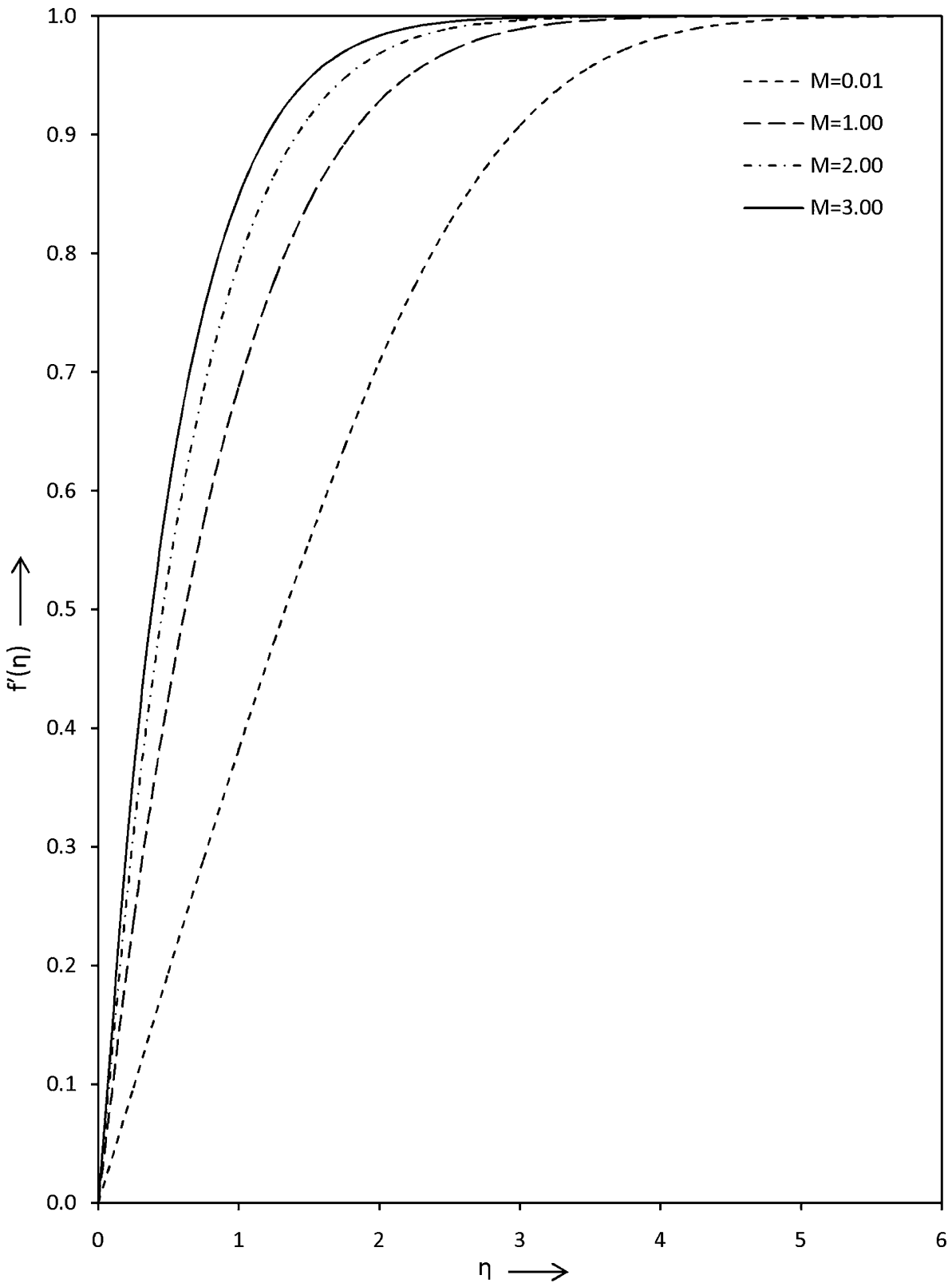

coefficient $C_{f}$ and the local Nusselt number $\mathrm{Nu}_{x}$ respectively. This table represents that the local skin friction coefficient $C_{f}$ decline with the sequence of nanofluids such as $\mathrm{Ag}$-water, $\mathrm{Cu}$-water, $\mathrm{TiO}_{2}$-water and $\mathrm{Al}_{2} \mathrm{O}_{3}$-water, although the local Nusselt number $N u_{x}$ evolves for the accrual manner of nanofluids like $\mathrm{Cu}$-water, $\mathrm{Ag}$-water, $\mathrm{TiO}_{2}$-water and $\mathrm{Al}_{2} \mathrm{O}_{3}$-water respectively. It is also clear from this table that the wall shear stress $f^{\prime \prime}(0)$ and the wall heat flux $\theta^{\prime}(0)$ boost for the booming values of the solid volume fraction $\phi$ and the magnetic parameter $M$. 
Fig. 8 Effect of $M$ with $C u$ water nanofluid on dimensionless temperature for $\phi=0.07$ $\operatorname{Pr}=6.2, B r=0.1$ and $B i=0.1$

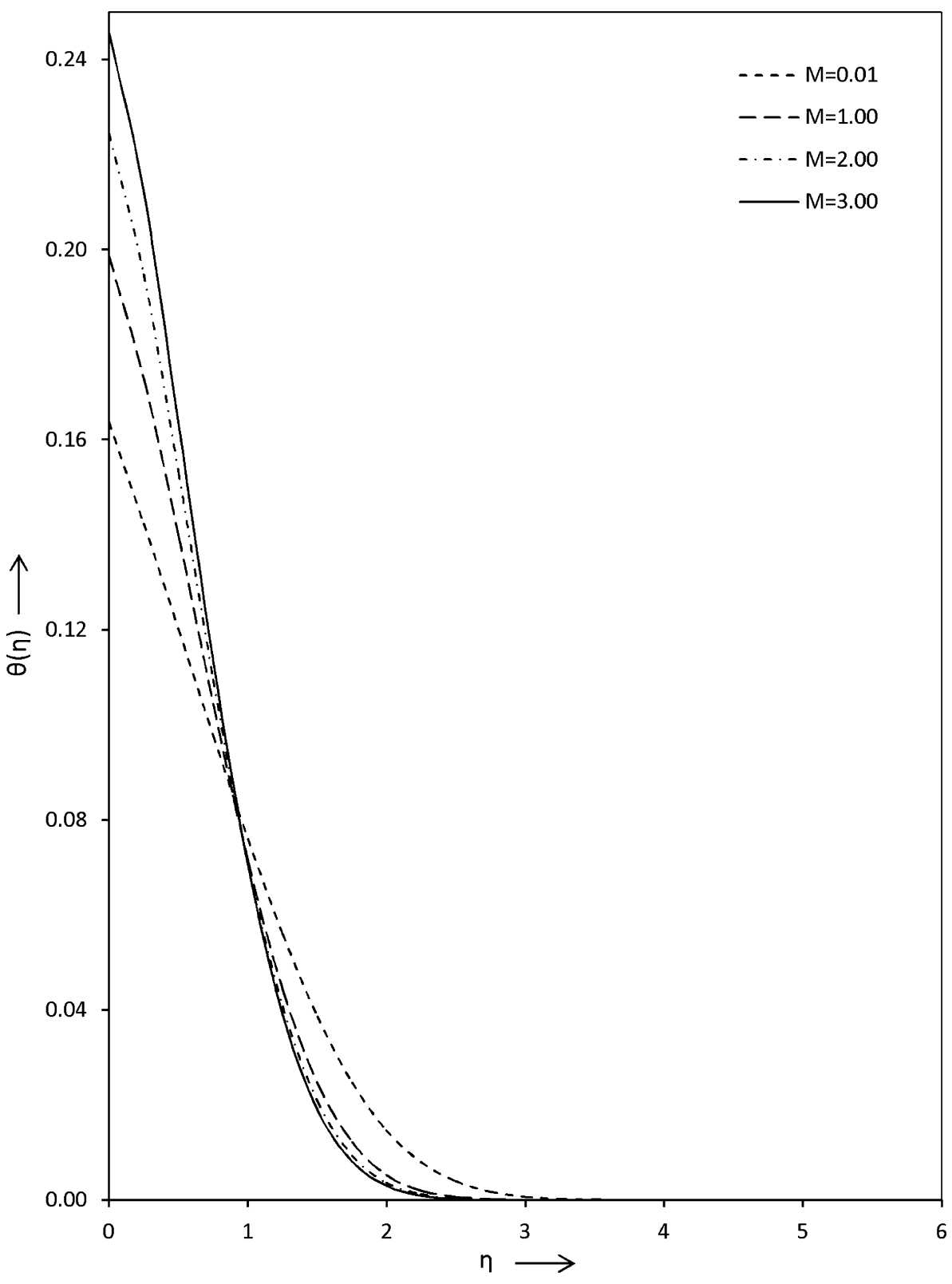

Further, increment in the Brinkmann number $\mathrm{Br}$ implies the enhancement in the local Nusselt number $\mathrm{Nu}_{x}$, while reverse trend is true for the local Biot number $\mathrm{Bi}$. Moreover, negative sign of the heat flux leads that there is a heat flow into the surface.

Finally, in the previous studies such as Chaudhary and Choudhary [8], and Chaudhary et al. [36], the magnetohydrodynamic flows of ordinary fluid have been examined and Galerkin finite element method applied to find the solution of the governing equations. Whereas the magnetohydrodynamic flow of various types of nanofluids have been explored by using the Keller-Box scheme in the present analysis. From these illustrations, it can be observed that nanofluids flow gives the comparatively better performance of the flow system than the ordinary fluids. 
Fig. 9 Effect of $\mathrm{Br}$ with $\mathrm{Cu}$ water nanofluid on dimensionless temperature for $\phi=0.07$, $M=0.01, \operatorname{Pr}=6.2$ and $B i=0.1$

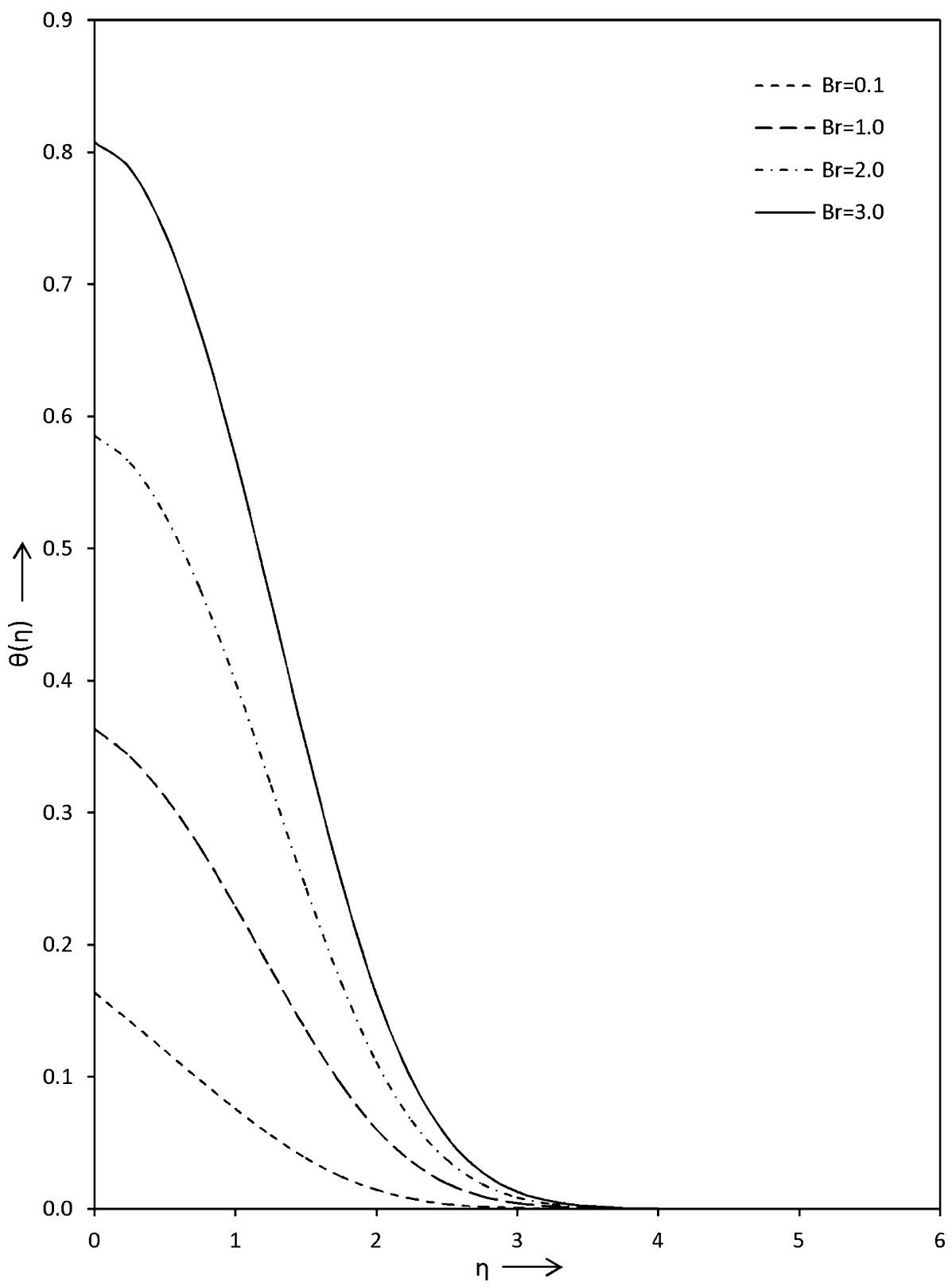

\section{Conclusions}

In the present analysis, a steady MHD nanofluids boundary layer flow past a flat surface with viscous dissipation and Joule heating influences along with the Newtonian heating is analyzed. The presence of four various kinds of nanoparticles namely $\mathrm{Ag}, \mathrm{Cu}, \mathrm{TiO}_{2}$ and $\mathrm{Al}_{2} \mathrm{O}_{3}$ with water based fluid is considered also. By using the similarity variables, the governing equations are converted into nondimensional form and solved by the Keller-Box method. 
Fig. 10 Effect of Bi with $\mathrm{Cu}$ water nanofluid on dimensionless temperature for $\phi=0.07$ $M=0.01, \operatorname{Pr}=6.2$ and $\mathrm{Br}=0.1$

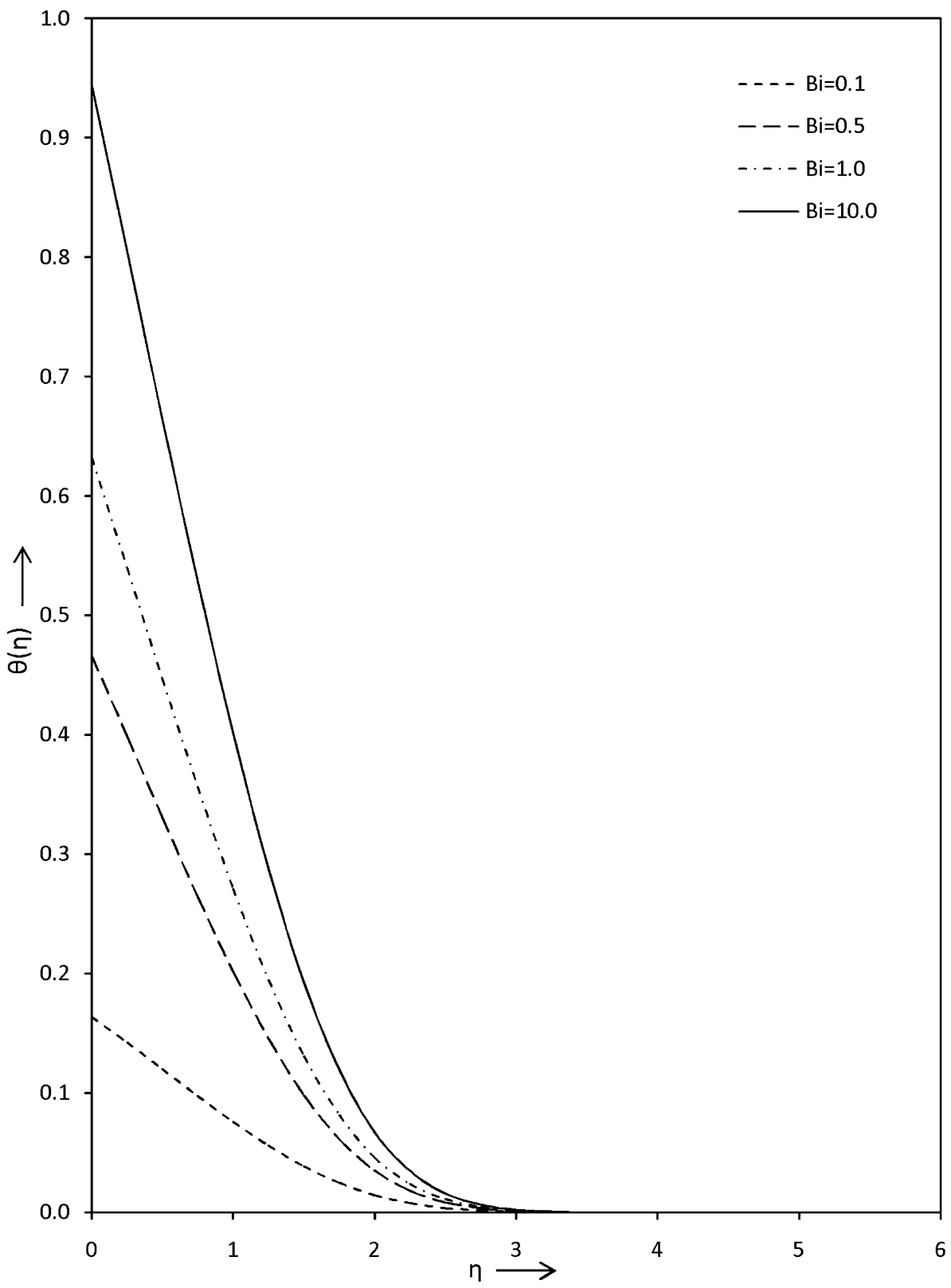

Moreover, the impacts of different nanofluids and pertinent parameters on the velocity, temperature, surface shear stress and the surface heat flux are examined and discussed. The main observations of present investigation are given as follows

(1) The velocity profile and the surface gradient are higher for Ag-water nanofluid than remaining nanofluids like $\mathrm{Cu}$-water, $\mathrm{TiO}_{2}$-water and $\mathrm{Al}_{2} \mathrm{O}_{3}$-water respectively. Whereas, $\mathrm{Al}_{2} \mathrm{O}_{3}$-water nanofluid has higher improvement on the temperature profile and the surface heat flux than other nanofluids.

(2) The momentum boundary layer, the thermal boundary layer, the local skin friction coefficient and the local Nusselt number increase as the solid volume fraction and the magnetic parameter develop, while opposite phenomenon occurs in the thermal bound- 
Table 3 Computed values of $f^{\prime \prime}(0)$ and $\theta^{\prime}(0)$ for distinct types of nanofluids and several values of specified parameters when $\operatorname{Pr}=6.2$

\begin{tabular}{|c|c|c|c|c|c|c|}
\hline Nanofluids & $\phi$ & $M$ & $B r$ & $B i$ & $f^{\prime \prime}(0)$ & $-\theta^{\prime}(0)$ \\
\hline Ag-water & 0.07 & 0.01 & 0.1 & 0.1 & 0.40348 & 0.0835793 \\
\hline Cu-water & & & & & 0.39077 & 0.0836163 \\
\hline $\mathrm{TiO}_{2}$-water & & & & & 0.35026 & 0.0834815 \\
\hline $\mathrm{Al}_{2} \mathrm{O}_{3}$-water & & & & & 0.34770 & 0.0833913 \\
\hline \multirow[t]{12}{*}{ Cu-water } & 0.01 & & & & 0.35439 & 0.0843381 \\
\hline & 0.04 & & & & 0.37516 & 0.0840001 \\
\hline & 0.10 & & & & 0.40207 & 0.0831959 \\
\hline & 0.07 & 1.00 & & & 1.06816 & 0.0801331 \\
\hline & & 2.00 & & & 1.46935 & 0.0775452 \\
\hline & & 3.00 & & & 1.78315 & 0.0754303 \\
\hline & & 0.01 & 1.0 & & 0.39077 & 0.0636384 \\
\hline & & & 2.0 & & & 0.0414407 \\
\hline & & & 3.0 & & & 0.0192430 \\
\hline & & & 0.1 & 0.5 & & 0.2668798 \\
\hline & & & & 1.0 & & 0.3675850 \\
\hline & & & & 10.0 & & 0.5566177 \\
\hline
\end{tabular}

ary layer for increment in the magnetic parameter when $\eta>1$.

(3) In case of enhancement in the Brinkmann number and the local Biot number, the fluid temperature as well as the heat transfer rate step-up, while reverse trend is happened in the heat transfer rate for the booming value of the local Biot number.

Funding This study was funded by Malaviya National Institute of Technology Jaipur (Grant Number F.4.R (Ph.D.) Acdm/ MNIT/2016/5499).

\section{Compliance with ethical standards}

Conflict of interest Authors declare that they have no conflict of interest.

\section{References}

1. El-Amin MF (2003) Combined effect of viscous dissipation and Joule heating on MHD forced convection over a non-isothermal horizontal cylinder embedded in a fluid saturated porous medium. J Magn Magn Mater 263:337-343

2. Abo-Eldahab EM, El-Aziz MA (2005) Viscous dissipation and Joule heating effects on MHD-free convection from a vertical plate with power-law variation in surface temperature in the presence of Hall and ion-slip currents. Appl Math Model 29:579-595

3. Jat RN, Chaudhary S (2009) Unsteady magnetohydrodynamic boundary layer flow over a stretching surface with viscous dissipation and Joule heating. II Nuovo Cimento 124B:53-59
4. Yavari H, Sadeghi A, Saidi MH, Chakraborty S (2012) Combined influences of viscous dissipation, non-uniform Joule heating and variable thermophysical properties on convective heat transfer in microtubes. Int J Heat Mass Transf 55:762-772

5. Das S, Jana RN, Makinde OD (2015) Magnetohydrodynamic mixed convective slip flow over an inclined porous plate with viscous dissipation and Joule heating. Alexandria Eng J $54: 251-261$

6. Hayat T, Imtiaz M, Alsaedi A (2016) Melting heat transfer in the MHD flow of $\mathrm{Cu}$-water nanofluid with viscous dissipation and Joule heating. Adv Powder Technol 27:1301-1308

7. Hussain A, Malik MY, Salahuddin T, Bilal S, Awais M (2017) Combined effects of viscous dissipation and Joule heating on MHD Sisko nanofluid over a stretching cylinder. J Mol Liq 231:341-352

8. Chaudhary S, Choudhary MK (2018) Finite element analysis of magnetohydrodynamic flow over flat surface moving in parallel free stream with viscous dissipation and Joule heating. Eng Comput 35:1675-1693

9. Alfven H (1942) Existence of electromagnetic-hydrodynamic waves. Nature 150:405-406

10. Ganesan P, Palani G (2004) Finite difference analysis of unsteady natural convection MHD flow past an inclined plate with variable surface heat and mass flux. Int J Heat Mass Transf 47:4449-4457

11. Jat RN, Chaudhary S (2010) Radiation effects on the MHD flow near the stagnation point of a stretching sheet. Z Angew Math Phys 61:1151-1154

12. Butt AS, Ali A (2014) Entropy analysis of magnetohydrodynamic flow and heat transfer due to a stretching cylinder. J Taiwan Inst Chem E 45:780-786

13. Imtiaz M, Hayat T, Alsaedi A, Hobiny A (2016) Homogeneous-heterogeneous reactions in MHD flow due to an unsteady curved stretching surface. J Mol Liq 221:245-253

14. Chaudhary S, Choudhary MK (2017) Viscous dissipation and Joule heating effects on an unsteady magnetohydrodynamic flow over a linearly stretching permeable surface with uniform wall temperature. Indian J Pure Appl Phys 55:864-872

15. Aydin SH, Selvitopi H (2018) Stabilized FEM-BEM coupled solution of MHD pipe flow in an unbounded conducting medium. Eng Anal Bound Elem 87:122-132 
16. Rehman, Ur K, Malik MY (2019) On Lie symmetry mechanics for Naviers-Stokes equations unified with non-Newtonian fluid model: a classical directory. Phys A Stat Mech Appl 122469

17. Rehman Ur K, Ali U, Zehra I, Malik MY, Ullah S (2019) Physical aspects of the Jeffery fluid inducing homogeneous-heterogeneous reactions in MHD flow: a Cattaneo-Christov approach. Can J Phys 999:1-7

18. Jha BK, Malgwi PB (2019) Combined effects of Hall and ion-slip current on MHD free convection flow in a vertical micro-channel. SN Appl Sci 1:1163

19. Choi SUS (1995) Enhancing thermal conductivity of fluids with nanoparticles. Publ Fed 231ASME, pp 99-106

20. Khanafer K, Vafai K, Lightstone M (2003) Buoyancy-driven heat transfer enhancement in a two-dimensional enclosure utilizing nanofluids. Int J Heat Mass Transf 46:3639-3653

21. Chein R, Chuang J (2007) Experimental microchannel heat sink performance studies using nanofluids. Int JTherm Sci 46:57-66

22. Yang YT, Lai FH (2010) Numerical study of heat transfer enhancement with the use of nanofluids in radial flow cooling system. Int J Heat Mass Transf 53:5895-5904

23. Mital M (2013) Semi-analytical investigation of electronics cooling using developing nanofluid flow in rectangular microchannels. Appl Therm Eng 52:321-327

24. Ibrahim W, Makinde OD (2015) Double-diffusive mixed convection and MHD stagnation point flow of nanofluid over a stretching sheet. J Nanofluids 4:28-37

25. Makinde OD, Khan WA, Khan ZH (2017) Stagnation point flow of MHD chemically reacting nanofluid over a stretching convective surface with slip and radiative heat. Proc Inst Mech Eng Part E J Process Mech Eng 231:695-703

26. Chaudhary S, Kanika KM (2019) Viscous dissipation and Joule heating in MHD Marangoni boundary layer flow and radiation heat transfer of $\mathrm{Cu}$-water nanofluid along particle shapes over an exponential temperature. Int J Comput Math. https://doi. org/10.1080/00207160.2019.1601713

27. Rehman Ur K, Shahzadi I, Malik MY, Al-Mdallal QM, Zahri M (2019) On heat transfer in the presence of nano-sized particles suspended in a magnetized rotatory flow field. Case Stud Therm Eng 14:100457

28. Kandasamy R, Atikah bt Adnan N, Radiah M, Kamarulzaki M (2019) Electric field strength on MHD aluminum alloys (AA7075) nanofluid flow. SN Appl Sci 1:12

29. Ma X, Sheikholeslami M, Jafaryar M, Shafee A, Nguyen-Thoi T, Li Z (2019) Solidification inside a clean energy storage unit utilizing phase change material with copper oxide nanoparticles. J Clean Prod 118888

30. Sheikholeslami M, Rezaeianjouybari B, Darzi M, Shafee A, Li Z, Nguyen TK (2019) Application of nano-refrigerant for boiling heat transfer enhancement employing an experimental study. Int J Heat Mass Transf 141:974-980
31. Merkin JH (1994) Natural convection boundary-layer flow on a vertical surface with Newtonian heating. Int J Heat Fluid Flow 15:392-398

32. Lesnic D, Ingham DB, Pop I, Storr C (2004) Free convection boundary-layer flow above a nearly horizontal surface in a porous medium with Newtonian heating. Heat Mass Transf 40:665-672

33. Makinde OD (2012) Computational modelling of MHD unsteady flow and heat transfer over a flat plate with Navier slip and Newtonian heating. Braz J Chem Eng 29:159-166

34. Akbar NS, Khan ZH (2015) Influence of magnetic field for metachoronical beating of cilia for nanofluid with Newtonian heating. J Magn Magn Mater 381:235-242

35. Hayat T, Bashir G, Waqas M, Alsaedi A (2016) MHD flow of Jeffrey liquid due to a nonlinear radially stretched sheet in presence of Newtonian heating. Results Phys 6:817-823

36. Chaudhary S, Kanika KM, Choudhary MK (2018) Newtonian heating and convective boundary condition on MHD stagnation point flow past a stretching sheet with viscous dissipation and Joule heating. Indian J Pure Appl Phys 56:931-940

37. Kamran M, Wiwatanapataphee B (2018) Chemical reaction and Newtonian heating effects on steady convection flow of a micropolar fluid with second order slip at the boundary. Euro J Mech B/Fluids 71:138-150

38. Vajravelu K, Prasad KV (2014) Keller-Box method and its application. Walter de Gruyter GmbH \& Co KG: Higher education press, Berlin

39. Makinde OD (2013) Effects of viscous dissipation and Newtonian heating on boundary-layer flow of nanofluids over a flat plate. Int J Numer Method Heat Fluid Flow 23:1291-1303

40. Bansal JL (1994) Magnetofluiddynamics of viscous fluids. Jaipur Pub House, Jaipur

41. Mohyud-Din ST, Khan U, Hassan SM (2016) Numerical investigation of magnetohydrodynamic flow and heat transfer of copperwater nanofluid in a channel with non-parallel walls considering different shapes of nanoparticles. Adv Mech Eng 8:1-9

42. Su X, Zheng $L$ (2013) Hall effect on MHD flow and heat transfer of nanofluids over a stretching wedge in the presence of velocity slip and Joule heating. Central Euro J Phys 11:1694-1703

43. Kumar R, Sood S (2016) Numerical analysis of stagnation point nonlinear convection flow through porous medium over a shrinking sheet. Int J Appl Comput Math. https://doi. org/10.1007/s40819-016-0150-2

Publisher's Note Springer Nature remains neutral with regard to jurisdictional claims in published maps and institutional affiliations. 